\title{
A Brief Solution to the Riemann Hypothesis over the Lagarias Transformation
}

\author{
Mesut KAVAK*
}

In accordance with the transformation of Lagarias [1] which is the equivalent of the Riemann Hypothesis, for a positive integer $n$, let $\sigma(n)$ denote the sum of the positive integers that divide $n$. Let $H_{n}$ denote the $n$th harmonic number by

$$
H_{n}=\sum_{n=1}^{n} \frac{1}{n}
$$

Does the following inequality hold for all $n \geq 1$ where $\sigma(n)$ is the sum of divisors function?

$$
H_{n}+\ln \left(H_{n}\right) e^{H_{n}} \geq \sigma(n)
$$

\section{Definition for the solutions}

Theorem: First of all, let's define an imaginary function as $\rho(n)$, and according to this function, assume that it becomes as the following.

$$
H_{n}=\frac{\sigma(n)+\rho(n)}{n}
$$

Here actually $\rho(n)$ is only by definition. There is no function like this and thus the rule of the function is not known. It is imaginary as a catalyzer. It does its work and leaves the actual functions alone without becoming inclusive when it shows us the result. This equation is only for relating $n, H_{n}$ and $\sigma(n)$ together somehow. $\rho(n)$ can be a negative number that is negative for the values of $\ln H_{n}>1$ here as we are going to see it over the below stated operations. If the result is suitable by the assumptions, then we can use it.

\section{Warning}

By using the equation, $H_{n}+\ln \left(H_{n}\right) e^{H_{n}} \geq \sigma(n)$ inequality turns into (1).

$$
H_{n}+\ln \left(H_{n}\right) e^{H_{n}} \geq n H_{n}-\rho(n)
$$

If it is edited, it becomes (2) over (2a).

$$
\begin{gathered}
\frac{\ln \left(H_{n}\right) e^{H_{n}}+\rho(n)}{n-1} \geq H_{n} \\
\ln \left(H_{n}\right) e^{H_{n}} \geq n H_{n}-H_{n}-\rho(n)
\end{gathered}
$$

Condition: Right this point assume, that the actual inequality is not (2) but is (3).

$$
\frac{e^{H_{n}}}{n} \geq H_{n}
$$

On (2), assume that actually the numerator is always bigger than $e^{H_{n}}$, and thus also if the divisor was $n-1$, this would increase the possibility of to be greater than $H_{n}$ of the division; so for the worst possibility, let's use this as (3). If the following operations are not verified over these above stated definitional assumptions, then we must redetermine the conditions and definitions.

Here if the numerator is bigger than $e^{H_{n}}$, then the equation becomes $\frac{\rho(n)}{1-\ln H_{n}}>e^{H_{n}}$ over $\ln \left(H_{n}\right) e^{H_{n}}+$ $\rho(n)>e^{H_{n}}$; so $\rho(n)$ is negative for $\ln H_{n}>1$.

\section{Warning}

Now, let (3) be (4).

$$
\sqrt[n]{e} \geq \sqrt[n H_{n}]{n H_{n}}
$$

$$
\begin{array}{r}
\text { For } e=\lim _{n \longrightarrow \infty}\left(1+\frac{1}{n}\right)^{n}, \text { (4) becomes (5). } \\
\qquad \lim _{n \longrightarrow \infty}\left(1+\frac{1}{n} \geq \sqrt[n H_{n}]{n H_{n}}\right)
\end{array}
$$

For this, it can be written as (6)

$$
\lim _{n \longrightarrow \infty}(n+1 \geq n k)
$$

where $k=\sqrt[n H_{n}]{n H_{n}}$. For $n \geq n k-1$ it becomes $\frac{1}{n} \geq k-1$; so what ever the direction of the inequality is, even if both sides were equal to each other, $k$ would not become a number smaller than 1 since $n$ is always positive. It is always $k>1$. Here assume, that is (7)

$$
n=n k-1+b
$$

since it is $n \geq n k-1$ over (6), where $b$ is a number being $b \in \mathbb{R}^{+}$ and thus being $b>0$; thus it becomes (8) over (7).

$$
n=\frac{b-1}{1-k}
$$

Since the inequality is $k>1$, then $b$ must always be smaller number than 1 to be positive of the division; thus it becomes $1>b>0$; so $k$ cannot take random values since $n$ is positive integer. If it is $k>1$, for the greatest value of $k$, it becomes $\lim _{b \rightarrow 0} k=2$. For this value, equality of (7) becomes $n=2 n-1$ and thus becomes $n=1$. It means, actually $k$ decreases as long 
as $n$ increased; thus it means it is always (9),

$$
1=\lim _{m \rightarrow \infty} \sqrt[m]{m}
$$

where $m \in \mathbb{Z}^{+}$; thus also means it is (10),

$$
1=\lim _{n \longrightarrow \infty} \sqrt[n H_{n}]{n H_{n}}
$$

since the inequality is $H_{n} \geq 1$ and thus is $n H_{n} \geq 1$.

\section{The Result: $\mathbf{R H}^{+}$}

By the above stated defined elements, in accordance with (6), if it is $\lim _{n \rightarrow \infty}(n+1 \geq n k)$, then it becomes $\lim _{n \rightarrow \infty}\left(\frac{1}{k-1} \geq\right.$ $n)$. Assume that it is actually $k=n-p$ where $p$ is real number. For this, the previous inequality becomes $\lim _{n \rightarrow \infty}\left(\frac{1}{n-p-1} \geq n\right)$ and thus becomes $\lim _{n \rightarrow \infty}\left(p \geq \frac{n^{2}-n-1}{n}\right)$. Here assume that actually for each $n$, the inequality always turns into $p=\frac{n^{2}-n-1}{n}$ equality. For this, $k=n-p$ becomes $k=\frac{n+1}{n}$ and thus (6) becomes (11).

$$
\lim _{n \rightarrow \infty}(n+1 \geq n+1)
$$

(11) shows us that for each $n, \lim _{n \rightarrow \infty}(n+1 \geq n k)$ inequality is defined; thus the above stated assumptions and imaginary functions are also suitable. Since (11) is also equivalent of (3), also it is equivalent of (12).

$$
H_{n}+\ln \left(H_{n}\right) e^{H_{n}} \geq \sigma(n)
$$

I again noticed, that the solution is not completely wrong, as also is not completely true. Namely if the numerator is bigger than $e^{H_{n}}$, then the inequality becomes $\frac{\rho(n)}{1-\ln H_{n}}>e^{H_{n}}$ over $\ln \left(H_{n}\right) e^{H_{n}}+\rho(n)>$ $e^{H_{n}}$; so $\rho(n)$ is negative for $\ln H_{n}>1$. It means we must take that imaginary function as negative for the definition at the beginning as

$$
H_{n}=\frac{\sigma(n)-\rho(n)}{n}
$$

After that the simplified inequality again becomes as the following,

$$
\frac{e^{H_{n}}}{n} \geq H_{n}
$$

where $n \geq 9$. Hence the rest is the same, and the following inequality holds for all $n \geq 9$ where $\sigma(n)$ is the sum of divisors function.

$$
H_{n}+\ln \left(H_{n}\right) e^{H_{n}} \geq \sigma(n)
$$

I do not know yet; but if it is accepted, (3) may be used over the Euler identity to develop new methods, for example for polar representation.

As a result, the result of $\mathrm{RH}$ is still $R H^{+}$. If also this is wrong, I promise I shall not work again forever about RH the calamitous problem.

Additionally I removed the definition of $\rho(n)$; because it remains non-sense, as it means different things as independent on the fiction. It could be anything.

Note on 11.11.2018

\section{Good bye!}

\section{References}

1. Jeffrey C. Lagarias. 2002 An Elementary Problem Equivalent to the Riemann Hypothesis, The American Mathematical Monthly. Vol. 109, No. 6, pp. 534-543 